\title{
Comparative chloroplast genomes of Paris Sect. Marmorata: insights into repeat regions and evolutionary implications
}

\author{
Xiaoyang Gao ${ }^{1}$, Xuan Zhang ${ }^{1,3}$, Honghu Meng ${ }^{2}$, Jing Li ${ }^{1,3}$, Di Zhang ${ }^{1,3}$ and Changning Liu ${ }^{1 *}$ \\ From 29th International Conference on Genome Informatics \\ Yunnan, China. 3-5 December 2018
}

\begin{abstract}
Background: Species of Paris Sect. Marmorata are valuable medicinal plants to synthesize steroidal saponins with effective pharmacological therapy. However, the wild resources of the species are threatened by plundering exploitation before the molecular genetics studies uncover the genomes and evolutionary significance. Thus, the availability of complete chloroplast genome sequences of Sect. Marmorata is necessary and crucial to the understanding the plastome evolution of this section and facilitating future population genetics studies. Here, we determined chloroplast genomes of Sect. Marmorata, and conducted the whole chloroplast genome comparison.

Results: This study presented detailed sequences and structural variations of chloroplast genomes of Sect. Marmorata. Over 40 large repeats and approximately 130 simple sequence repeats as well as a group of genomic hotspots were detected. Inverted repeat contraction of this section was inferred via comparing the chloroplast genomes with the one of $P$. verticillata. Additionally, almost all the plastid protein coding genes were found to prefer ending with A/ $U$. Mutation bias and selection pressure predominately shaped the codon bias of most genes. And most of the genes underwent purifying selection, whereas photosynthetic genes experienced a relatively relaxed purifying selection.

Conclusions: Repeat sequences and hotspot regions can be scanned to detect the intraspecific and interspecific variability, and selected to infer the phylogenetic relationships of Sect. Marmorata and other species in subgenus Daiswa. Mutation and natural selection were the main forces to drive the codon bias pattern of most plastid protein coding genes. Therefore, this study enhances the understanding about evolution of Sect. Marmorata from the chloroplast genome, and provide genomic insights into genetic analyses of Sect. Marmorata.
\end{abstract}

Keywords: Paris Sect. Marmorata, Chloroplast genome, Repeat sequence, Codon usage, Evolutionary rates

\section{Background}

Herbal medicine is currently becoming increasingly popular to be used in complementary and alternative treatments all over the world. Moreover, herbal medicine is still a major source of healthcare, especially in developing countries, which have limited access to modern medical care [1]. However, the wild resources

\footnotetext{
* Correspondence: liuchangning@xtbg.ac.cn

${ }^{1}$ CAS Key Laboratory of Tropical Plant Resources and Sustainable Use, Xishuangbanna Tropical Botanical Garden, Chinese Academy of Science, Menglun 666303, Yunnan, China

Full list of author information is available at the end of the article
}

of plant species are threatened by plundering exploitation with the population growth, particularly the increasing demand for herbal medicine with significant economic value.

The species of Paris are famous herbal essence for the elements like steroidal saponins with effective pharmacological therapy. Here, we selected the rare species of the genus Paris Section Marmorata H. Li to explore the chloroplast genome analyses. The Sect. Marmorata comprises two species of perennial medicinal herbs, i.e., P. marmorata Stearn and $P$. luquanensis H. Li. P. marmorata is mainly distributed

(c) The Author(s). 2018 Open Access This article is distributed under the terms of the Creative Commons Attribution 4.0 International License (http://creativecommons.org/licenses/by/4.0/), which permits unrestricted use, distribution, and reproduction in any medium, provided you give appropriate credit to the original author(s) and the source, provide a link to the Creative Commons license, and indicate if changes were made. The Creative Commons Public Domain Dedication waiver (http://creativecommons.org/publicdomain/zero/1.0/) applies to the data made available in this article, unless otherwise stated. 
in Southwest China (i.e., Yunnan, Sichuan, and Tibet), Nepal, and Bhutan, while P. luquanensis is mainly distributed throughout Yunnan (i.e., Luquan and Pingbian) and Sichuan (i.e., Huidong, Puge and Yuexi) [2]. Their morphological characteristics are different from other Paris members, as these two species have variegated leaves, grow more slowly and thus are shorter than other Paris plants. As the other plants of Paris, Sect. Marmorata species have been used in oriental medicine for a long time. They contain Rhizoma Paridis saponins including diosgenyl and pennogenyl saponins as active ingredients, which are typically used in the treatment of tumors, hemostasis, and inflammation [3-5]. Thus, wild resources of $P$. marmorata and $P$. luquanensis are rapidly declining as consequence of their slow growth and low levels of artificial cultivation, but overexploitation for the economic value. What is more serious is that the wild plants are hard to find, but little is known about the sequence diversity and structure divergence of their chloroplast genomes.

Chloroplasts are essential plant organelles that originated from Cyanobacteria by endosymbiosis with the precursor of a nucleated ancestral cell more than 1.2 billion years ago [6]. The circular, double stranded chloroplast genome encodes a set of proteins involved in photosynthesis and other biochemical pathways that are important for plant growth and development, even plant evolution, such as biosynthesis of starch, fatty acids, and pigments [7]. Chloroplast genomes of plants are known to be predominantly uniparentally inherited and highly conserved in both gene order and gene contents [8]. They typically have a quadripartite organization, consisting of two IR regions separated by two regions of unique DNA, LSC region and SSC region [9]. Substitution rates of chloroplast genomes are much lower than those of nuclear DNA, which are even more substantially reduced in the IR regions [10]. Low levels of recombination and primarily uniparental inheritance make chloroplast genomes a valuable source of genetic markers for phylogenetic analyses and useful tools for DNA barcoding [11, 12]. High proportion of SSRs has aroused considerable interest due to the ability to generate highly informative DNA markers [13]. In the light of higher levels of allelic variation of SNPs, SSRs make their use as indicators for species identification, hybridization and introgression analyses [13-15], and they have been widely applied to investigating population differentiation and other plant science studies [16-19]. Therefore, genome-wide comparative analysis of SSRs distribution in chloroplast genomes will lay the foundation for further monitoring gene flow, population differentiation and cytoplasmic diversity of Paris plants with intricate hybridization.
Previous study was focus on the phylogeny of Paris [20], but lacking of detailed information about genetic variation and molecular structural diversity in these species. The structural and nucleotide sequence variations among chloroplast genomes of Sect. Marmorata can be exposed by combining the chloroplast genomes of this section aligned to different reference chloroplast genomes (e.g., P. verticillata and P. polyphylla var. yunnanensis). Thus, chloroplast genomes of Sect. Marmorata were sequenced using the Illumina sequencing platform. The comparative analyses of chloroplast genomes will contribute to further investigating genetic diversity and evolution of this section to support conservation management strategies, and assist in the exploration and utilization of Paris Sect. Marmorata in herbal medicine.

Herein, the aims of this study enable us: (1) to examine sequence variations and screen for hotspot regions in Sect. Marmorata chloroplast genomes; (2) to characterize global structural patterns of chloroplast genomes of Sect. Marmorata; and (3) to explore codon usage patterns and substitution rates of protein coding genes from chloroplast genomes of Sect. Marmorata species.

\section{Results and discussion}

Chloroplast genome assembly, organization, and features Illumina paired-end sequencing produced $692 \mathrm{Mb}$ and $762 \mathrm{Mb}$ of data from chloroplast genomes of $P$. marmorata and $P$. luquanensis, respectively. P. marmorata, 1,024,696 reads of a total of 1.54 million were mapped to the reference chloroplast genome with a mean depth of $1,028 \times$. And P. luquanensis, 1,662,396 reads of a total of 1.68 million were mapped to the reference chloroplast genome with a mean depth of $1,120 \times$. The N50 contigs length for $P$. marmorata and $P$. luquanensis were $6,990 \mathrm{bp}$ and $36,582 \mathrm{bp}$, and the complete chloroplast genome sequences were 157,629 bp and 157,643 bp in length, respectively. As the other angiosperms, chloroplast genomes of Sect. Marmorata had a typical quadripartite structure consisting of a pair of identical IRs $(27,521-27,611 \mathrm{bp})$, separated by LSC (84,028-84,059 bp) and SSC (18,393-18,529 bp) regions (Fig. 1; Additional file 1: Table S1).

The chloroplast genomes of Sect. Marmorata encoded an identical set of 133 predicted functional genes, 113 of which were unique, and 20 were duplicated and located in the IR regions. The 113 unique genes were comprised of 30 tRNA genes, 4 rRNA genes, and 79 protein-coding genes, respectively (Additional file 1: Table S1). Fifteen distinctive genes, including $a p t F, n d h A$, pet $B, r p l 2$, and $\operatorname{trn} A-U G C$, contained the single introns; while the genes clpP, rps12, and $y c f 3$ contained two introns (Additional file 1: 


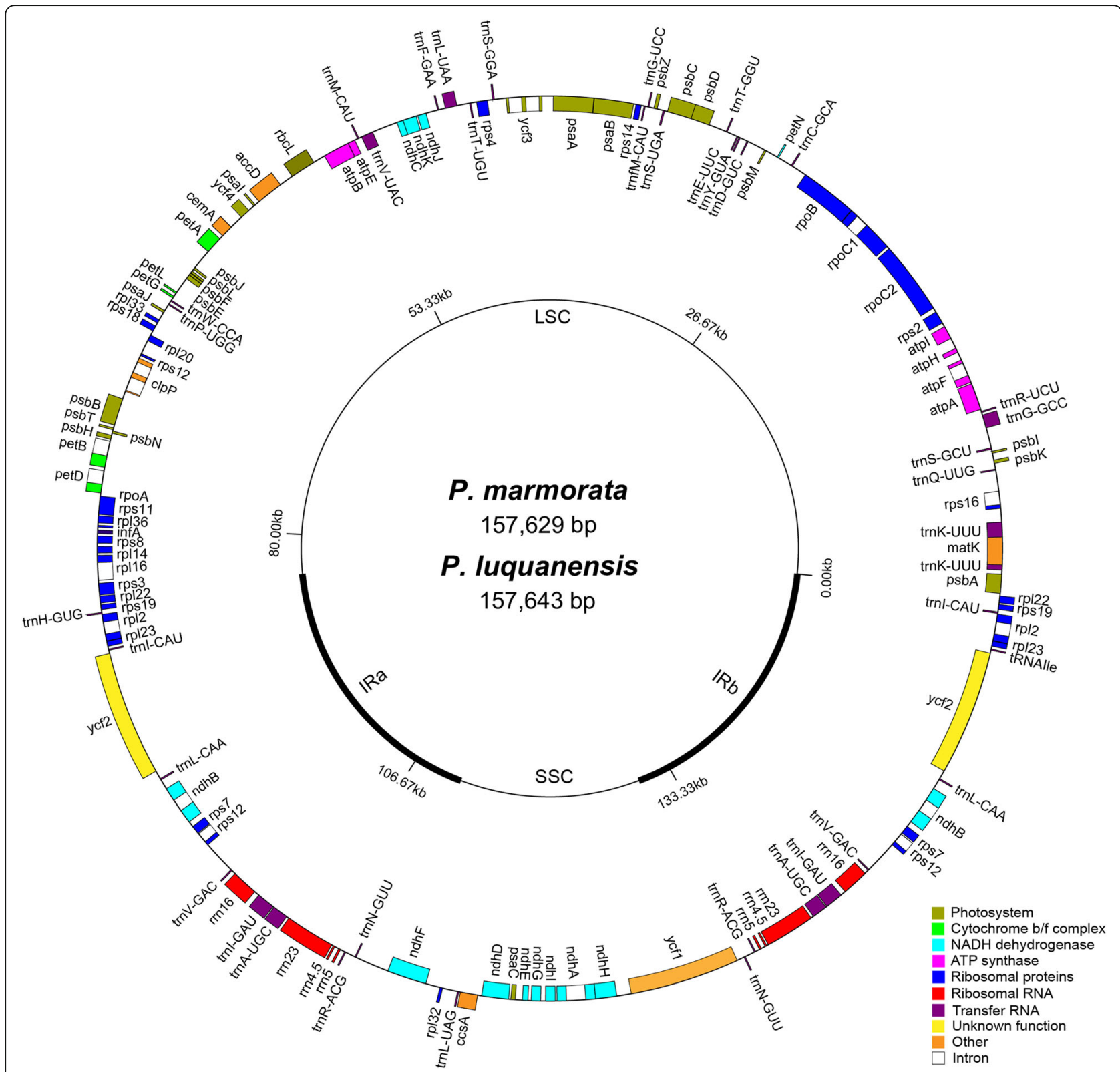

Fig. 1 Gene map of chloroplast genomes of Paris Sect. Marmorata, P. marmorata and P. luquanensis. Genes belonging to different functional categories are color-coded. Genes shown the inside circle are transcribed clockwise and those located on the outside are transcribed counterclockwise

Table S2). These introns share the same splicing mechanism as group II introns [21]. However, different assembly strategies and different reference chloroplast genomes (i.e., $P$. verticillata versus $P$. polyphylla var. yunnanensis) led to some differences in gene contents. In the light of sequence similarity, the chloroplast genome of P. polyphylla var. yunnanensis was regarded as the most similar genome for Sect. Marmorata via BLAST in NCBI. In addition, Sect. Marmorata species and P. polyphylla var. yunnanensis are closely related, and they belong to the subgenus Daiswa. Notably, comparison of gene contents revealed that two genes were unique to chloroplast genomes sequenced in this study, and another four genes were unique to chloroplast genomes sequenced by Huang et al, respectively (Additional file 2: Figure S1).

A total of $59.41-60.03 \%$ of Sect. Marmorata chloroplast genomes were protein-coding regions. Overall, $1.83 \%, 5.74 \%$, and $52.02-52.73 \%$ of the genome sequences encoded tRNAs, rRNAs, and proteins, respectively. The remaining sequences consisted of noncoding regions filled with introns, intergenic spacers, and a pseudogene. The $y c f 1$-like $\left(y c f 1^{\Psi}\right)$ gene in the IRb/SSC junction was found to be the only pseudogene, resulting 
from an incomplete duplication of the normal functional copy of $y c f 1$ in the IRa/SSC junction. Similar to the chloroplast genomes of $P$. verticillata and $P$. polyphylla var. yunnanensis, the chloroplast genomes of Sect. Marmorata were AT-rich with an overall AT content of 62.6\%. AT content of LSC (64.25-64.28\%), SSC (68.70$70.10 \%)$, and IR (58.08-61.10\%) regions varied slightly. AT content of genomic regions is probably associated with dynamics of repeat sequences and codon bias of chloroplast protein-coding genes [22, 23]. In general, the chloroplast genome features of Sect. Marmorata were similar in the terms of gene content, gene order, introns, intergenic spacers, and AT content.

\section{Repeat sequence analyses}

Repeat regions play an important role in recombination and genomic rearrangements [24, 25]. Eleven sets of repeats were identified in chloroplast genome sequences of $P$. marmorata and P. luquanensis using TRF with a $100 \%$ match criterion in repeat copies. With a $>90 \%$ match criterion, another 10 sets of repeats were identified, yielding 21 total sets detected in P. marmorata, with 12 in CDS regions, 8 in intergenic regions and 1 in a span spacer of a CDS region. Similarly, with a > 90\% match criterion, another 8 sets of repeats, yielding 19 total sets were detected in P. luquanensis, with 9 in CDS regions, 9 in intergenic regions, and 1 in a span spacer of tRNA. The repeats were scattered around the LSC (6-7), SSC (6-8), and IRs (6-7) regions, and they mainly located in the intergenic regions and protein coding regions, including $a c c D, r b c L, y c f 1$ and $y c f 2$ (Additional file 1: Table S3).

Meanwhile, a total of 30 repeats were identified in chloroplast genome sequences of both $P$. marmorata and $P$. luquanensis, and the sizes of repeats ranged from 63 to $139 \mathrm{bp}$. Those repeats were scattered around SSC and IRs regions, and they mainly located in the intergenic regions, protein coding regions $(y c f 1$ and $y c f 2$ ) and $y c f 1^{\Psi}$ (Additional file 1: Table S4). There were 14 and 21 repeats with 0 hamming distance in $P$. marmorata and $P$. luquanensis, respectively, that is, these were repeats with $100 \%$ identity. The output of REPuter was compared with the one of TRF, and the tandem repeats and dispersed repeats (i.e., forward and palindromic) were separately analyzed. The total numbers of those repeats were 51 and 49 for $P$. marmorata and $P$. luquanensis, respectively, in which their copy numbers ranged from 2 to 16 . Among the coding regions, the richest in repeats was the $y c f 1$ gene, which contained 31 and 30 repeats in P. marmorata and P. luquanensis, respectively. As reported in the other chloroplast genomes, $y c f 2$ was also rich in repeats, carrying 4 to 5 repeats. These two protein-coding genes and divergent regions are demonstrated to be often associated with repeat events [26]. The above-mentioned repeats can provide valuable information on developing markers for phylogenetic research and population studies.

SSRs are stretches of small repeating units of DNA occurring in both coding and non-coding regions. SSRs have been used as DNA markers for population genetic studies, due to their polymorphic nature and co-dominant expression [27]. Chloroplast SSRs have been commonly used to characterize genetic variation among plant genotypes [28-31]. In chloroplast genomes of Sect. Marmorata, 128-130 SSRs were identified in silico, of which, 59-62 were mononucleotides, 22-26 were dinucleotides, 8-9 were trinucleotides, 20 were tetranucleotides, 7 were pentanucleotides, and 8-10 were hexanucleotides (Additional file 1: Table S5). A total of 22 and 21 SSRs for P. marmorata and P. luquanensis, respectively, occurred in compound formations that comprised several combinations of SSRs separated by the maximum distance of $100 \mathrm{bp}$. The most abundant motifs were mononucleotide $\mathrm{A} / \mathrm{T}$ repeats (contributed to $96.60 \%$ of mon-repeat SSRs), comprising about $43.85-46.88 \%$ of total repeats. The distribution pattern of six SSR motifs in Sect. Marmorata was consistent with that of other Paris species (Additional file 2: Figure S2). Gene regions harbored more SSRs (over 40 SSRs) than gene spacer did, and protein coding genes including rps12, $y c f 1$, etc., harbored more than two SSRs. The length of most SSRs (over 90\%) ranged from $10 \mathrm{bp}$ to $19 \mathrm{bp}$ (Fig. 2a; Additional file 3: Table S6). Repeat times of mononucleotide repeats were mostly over 9 , and repeat times of most rest five motifs were less than 9 (Fig. 2b). Chloroplast genomes contain conserved genes, but the number of SSRs that they harbor varied. Compared to IR regions, the LSC and SSC regions contain more SSRs, and this finding is consistent with analyses of other angiosperm chloroplast genomes [32-34]. In order to identify whether they are efficient markers for delineating Sect. Marmorata, those chloroplastic microsatellites were assessed in silico by calculating Shannon-Winener index and PIC. SSRs in gene regions, such as $y c f 1$, etc.; and SSRs in gene spacers such matK-rps16, etc., showed high polymorphism (PIC $>0.5$ ), which can be exploited for genetic diversity studies of Sect. Marmorata (Additional file 3: Table S7). Moreover, the inferred phylogenetic trees revealed that Sect. Marmorata had a well-supported consistent topology, in the light of phylogenetic analyses of some genes and spacers harbored repeat sequences. The regions like $y c f 1-n d h F$ etc., can be the source of genetic markers, assisting in phylogeny and population studies of Sect. Marmorata. However, the other sections of the subgenus Daiswa showed unstable topologies and the relationships among them were still not very clear, due to lacking of enough representatives of Paris (Additional file 2: Figure S3). 

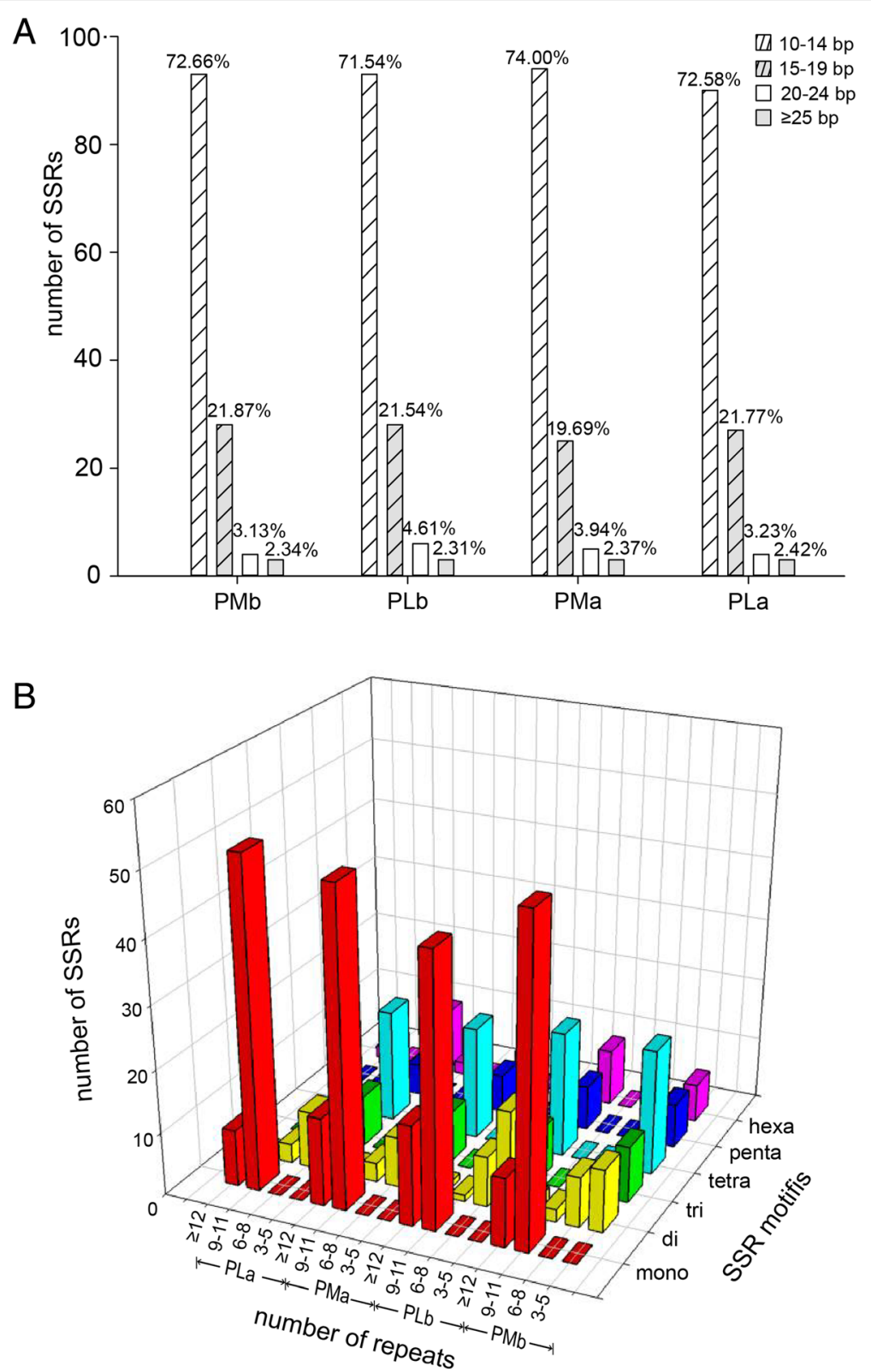

Fig. 2 Distribution of microsatellites in chloroplast genomes of Sect. Marmorata. a length distribution of SSRs in four chloroplast genomes of Sect. Marmorata; $\mathbf{b}$ distribution of chloroplast SSRs repeat number with respect to motif type

\section{Chloroplast genome comparison of sect. Marmorata}

The availability of complete chloroplast genomes from the genus Paris provides an opportunity to elucidate the chloroplast genome organization and sequence variation of Sect. Marmorata. Sequence divergence analyses of four chloroplast genomes of Sect. Marmorata revealed $\pi$ values in the range from 0 to 0.01639 with an average of 0.0009 , indicating there are a few differences among the four chloroplast genomes (Fig. 3). However, eight protein coding regions (including $p s a A$, $a c c D, p s b E, p s b B, r p s 19, y c f 1, y c f 2$, and $r p l 2)$, and seven intergenic regions (including psbE-petL, psbB-psbT,

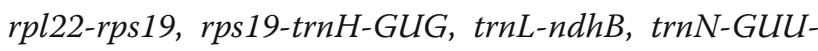
trnR-ACG, and trnR-rrn5) as well as the pseudogene $y c f 1^{\Psi}$ had higher divergence values $(\pi>0.004)$, indicating that they harbored more variations than other regions. This is in accordance with the previous study, as a lot of SNPs were detected in the above regions of other Paris chloroplast genomes [20]. These regions with highly diverse loci are not random, but they are instead clustered in "hot spots" [35, 36].

The overall sequence identities of four chloroplast genomes of Sect. Marmorata were plotted using mVISTA and the chloroplast genome of $P$. verticillata 


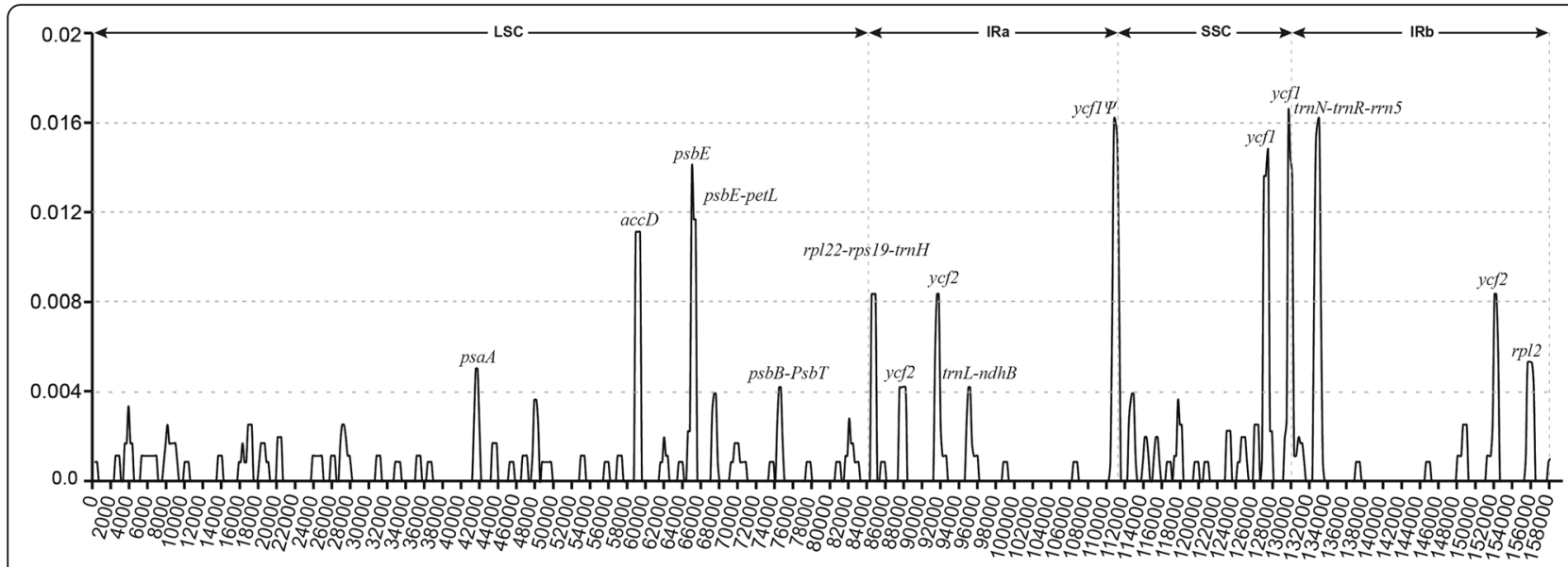

Fig. 3 Sliding window analysis of the whole chloroplast genome of Sect. Marmorata. X-axis, position of midpoint of a window; Y-axis, nucleotide diversity of each window

was used as a reference (Fig. 4). The IR regions showed lower sequence divergence than the LSC and SSC regions, possibly due to copy number differences in IR sequences caused by gene conversion [37]. As expected, non-coding regions exhibited higher sequence divergence than coding counterparts, and the most divergent non-coding regions in the chloroplast genomes located in the intergenic spacers. Intergenic regions with high levels of divergence included atpH/atpI and trnH-GUG/psbA. The most divergent coding regions were $y c f 1$, $a t p F$, and $a c c D$. Accordingly, universal primers for these intergenic regions could aid in phylogenetic inference and population genetic analysis of Sect. Marmorata.

Expansion and contraction of IR regions are common in the evolutionary history of land plants. Indeed, the junctions of LSC/IR and IR/SSC are sometimes regarded as an index of chloroplast genome evolution [38]. To evaluate the potential impact of the junction changes in the chloroplast genomes of Sect. Marmorata, we compared the IR boundaries of Sect. Marmorata species with that of their references (Fig. 5). In those analyzed chloroplast genomes, IR/SSC boundary was located within the $y c f 1$ gene, resulting in the formation of $y c f 1$ pseudogene. $y c f 1$ observed in subgenus Daiswa (Sect. Marmorata species and P. polyphylla var. yunnanensis) was longer than its homologue in the subgenus Paris ( $P$. verticillata). Additionally, the LSC/IR junction was detected a contraction event in subgenus Daiswa (expect for chloroplast genomes of P. marmorata and $P$. luquanensis sequenced by Huang et al., namely PMa and PLa). As seen in other chloroplast genomes studies [39], contraction of IR regions in Sect. Marmorata has led to changes in the chloroplast genome structure, causing the formation of pseudogenes. It is worth noting that obvious differences in the LSC/IR boundary could also be observed among Sect. Marmorata species, because of the different reference chloroplast genomes and assembly methods adopted in the two studies. LSC/IR boundaries of PMa and PLa were next to rps19 and they were found to be more similar to that of Veratrum patulum in the family Melanthiaceae [40]. More sequences are required to further analyze and confirm the IR boundaries of chloroplast genomes of the section. The above analyses also implied that length differences in noncoding regions could affect genome size variation among species of Sect. Marmorata.

\section{Codon usage pattern}

Most protein-coding genes employed the standard initiator codon AUG; however, six unusual start codons were also identified, such as TTG (cemA) and GTG (rps19). Similar noncanonical start codons have been detected in other angiosperms and tree fern plants [4143]. Furthermore, the codon usage patterns were determined for 71 distinct protein-coding genes in Sect. Marmorata chloroplast genomes. Codons of chloroplast genes of Sect. Marmorata with A/U at the third position nucleotide were used more frequently than those ending with $\mathrm{G} / \mathrm{C}$, according to RSCU values (with a threshold of RSCU >1). As observed in chloroplast genomes of most land plants, codon usage patterns of this section are likely driven by the composition bias towards the high $\mathrm{A} / \mathrm{T}$ content.

The ENc plots are useful indicators for the factors affecting codon bias. To determine the relative importance of mutation and selection in producing codon usage patterns, ENc values were estimated and plotted against the GC3s values (Fig. 6). The protein-coding genes from four chloroplast genomes of Sect. Marmorata species shared the analogous codon bias patterns. A small number of genes from 


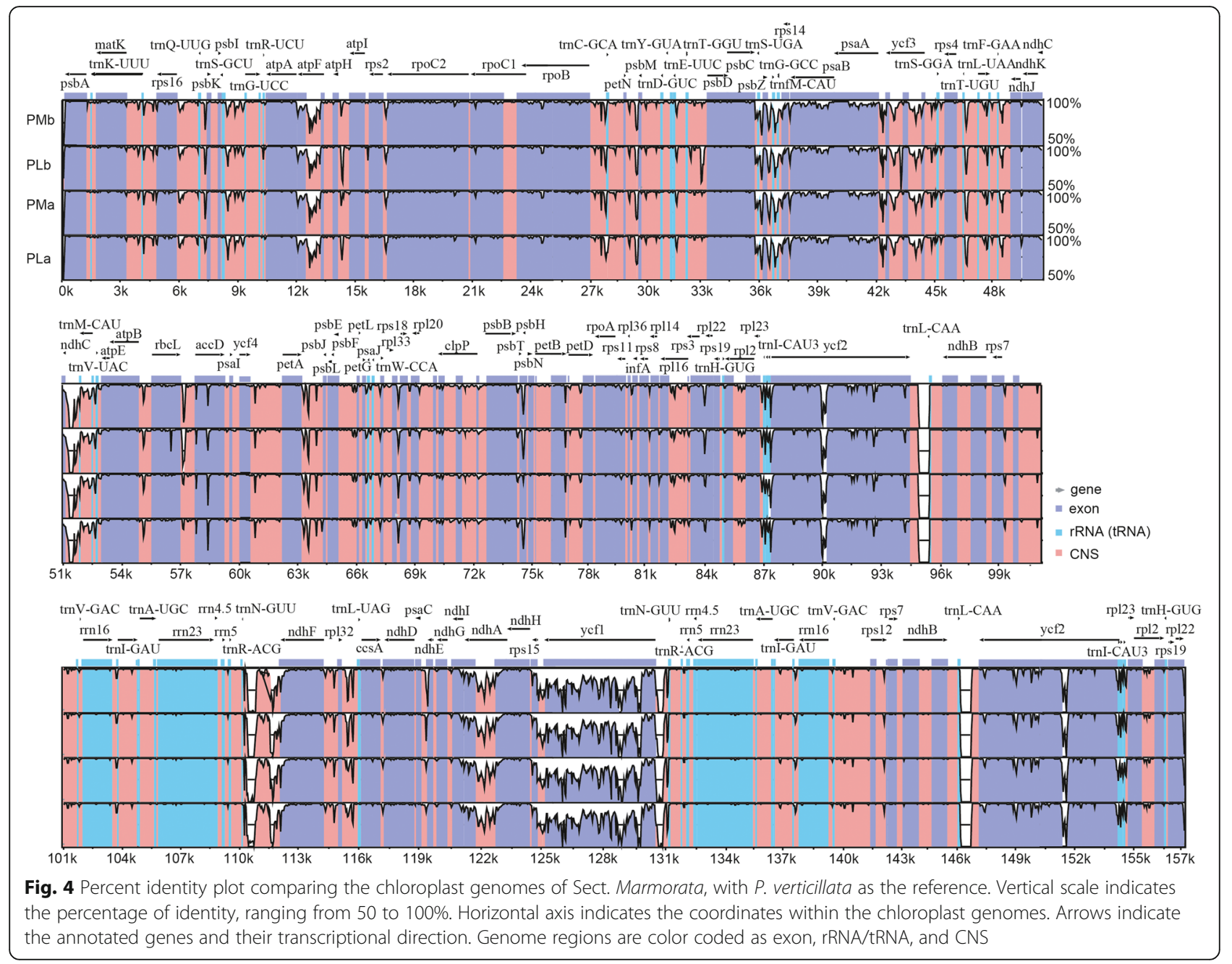

three functional categories exactly followed the standard curve, which certainly originated from extreme GC compositional constraints, and their codon biases were consistent with by mutation bias [44]. In particular, more than half of genes were below the curve, suggesting that the selection forces predominantly influenced these genes, and that natural selection had more influence on codon bias of chloroplast genome [45]. Intriguingly, genes associated with photosynthesis showed a discrete distribution, which implied that other factors such as gene length and gene expression level also influenced the codon bias.

\section{Evolutionary rates of sect. Marmorata chloroplast genes}

$K_{\mathrm{a}}, K_{\mathrm{s}}$ and $K_{\mathrm{a}} / K_{\mathrm{s}}$ are valuable indicators of evolutionary rates and natural selection [46]. To better understand the evolutionary forces shaping chloroplast genes of Sect. Marmorata, the substitution rates $\left(K_{\mathrm{a}}\right.$ and $K_{\mathrm{s}}$, and $K_{\mathrm{a}} / K_{\mathrm{s}}$ ratios) of 77 conserved protein-coding genes were estimated using $P$. verticillata as reference (Additional file 2: Figure S4). Overall, chloroplast protein-coding genes from different Sect. Marmorata species had similar substitution rates. However, significant high $K_{\mathrm{a}} / K_{\mathrm{s}}$ ratios of gene $p s b B, r p l 20$ and rps 2 from $P$. luquanensis sequenced simultaneously (PLa) were discovered, which were obviously different from those values of P. luquanensis sequenced in this study (PLb), and $P$. marmorata sequenced simultaneously (PMa) and $P$. marmorata sequenced in this study $(\mathrm{PMb})$. And the $K_{\mathrm{a}} / K_{\mathrm{s}}$ values of most protein-coding genes were less than 1 , except for rpoC1, rps11, accD, $c l p P$, and $y c f 2$, which indicated that most of the genes experienced a purifying selection. The $K_{\mathrm{a}} / K_{\mathrm{s}}$ ratios of protein-coding genes in the IR regions were higher than those in LSC and SSC regions. Additionally, a set of genes with $K_{\mathrm{a}} / K_{\mathrm{s}}$ values exceeding 45 or $K_{\mathrm{a}} / K_{\mathrm{s}}$ values that were otherwise incalculable (NA), included rps7, rps19, and rps12 in the IR region (with $K_{\mathrm{a}} / K_{\mathrm{s}}$ values exceeding 50 ), aptI, psaI, psbF, psbN, rpl16, rpl20, rps18, rps2, and rps4 in LSC region (with $K_{\mathrm{a}} / K_{\mathrm{s}}$ values exceeding 45), and petL, pet $N$, ndhJ, psaJ, psbI, psbJ, psbL, rpl33, rpl36, rps16, ndhE, and rpl32 (Ka/Ks values were "NA"). High and incalculable values 

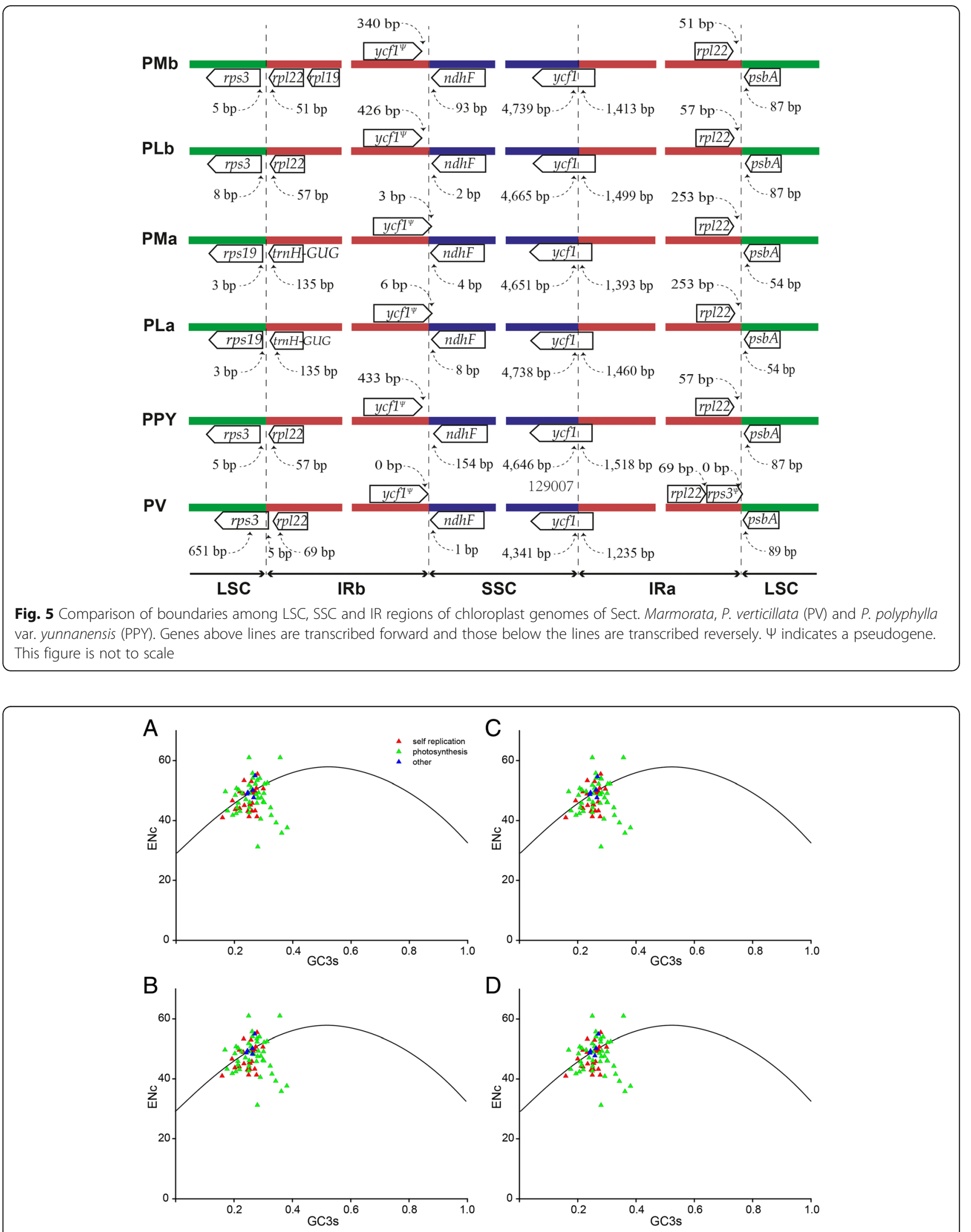

Fig. 6 ENc plotted against GC3s. The solid line represents the expected curve of positions of genes when the codon usage was only determined by the GC3s composition. ENc and GC3s plots for Sect. Marmorata: a PMa, b PMb, c PLa, d PLb 
occurred when the $K_{\mathrm{s}}$ values were extremely low or when there were no substitutions in the alignment (i.e., there was a $100 \%$ match), respectively. For both cases, the values of NA and ratios over 45 were manually changed to 0 [47]. After sorting the genes into functional categories and groups, widely variable substitution rates among 77 genes were discovered (Fig. 7; Additional file 2: Figure S5). $K_{\mathrm{a}}$ and $K_{\mathrm{a}} / K_{\mathrm{s}}$ of genes related to photosynthesis were apparently lower than that of genes related to self-replication as well as other types of genes. It can speculate that genes involved in photosynthesis tend to be more divergent than genes related to self-replication and other genes, and photosynthetic genes are under somewhat relaxed purifying selection [47]. Although the causes and consequences of evolutionary rate

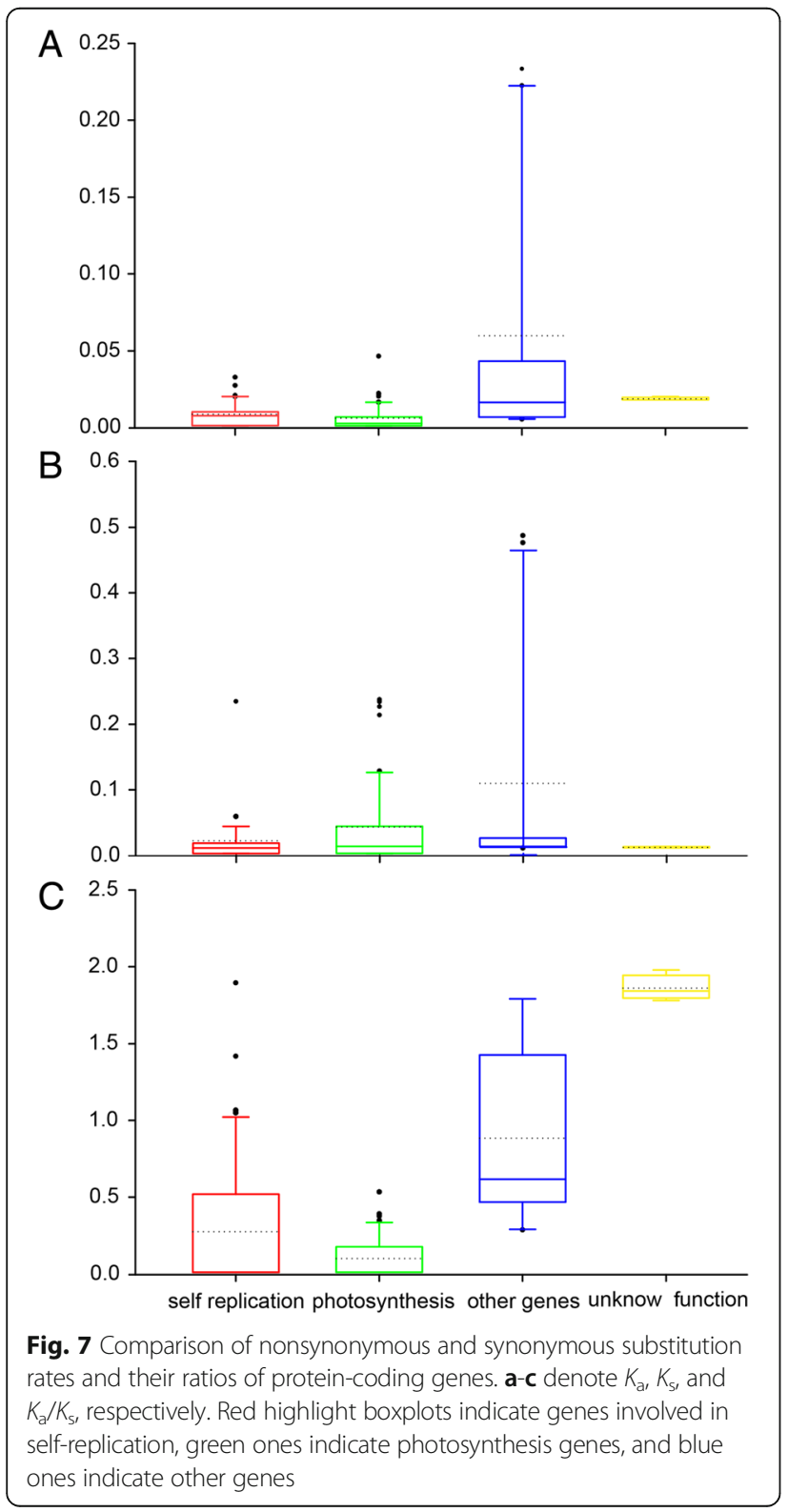

differences among protein-coding genes remain a subject of debate, disparities in generation time, relaxed selection, length of encode protein products, gene expression level, and gene function have all been suggested as potential explanations of such differences [48-51].

\section{Conclusions}

Complete chloroplast genome sequences of Sect. Marmorata, i.e., P. marmorata and P. luquanensis were assembled, annotated and explored subsequent genome-wide comparative analyses. The chloroplast genomes exhibit a typical quadripartite structure of LSC and SSC regions separated by a pair of IRs, and they share similar features in the terms of gene organization and AT-rich content. Large repeats, polymorphic SSR loci, as well as genes and intergenic regions with high levels of variability were determined. Those repeat motifs and hotspot regions can be selected to study both intraspecific and interspecific variability, and they also can aid in inferring phylogenetic relationships of Sect. Marmorata and other species of subgenus Daiswa. Non-coding regions like some intergenic spacers exhibited significantly higher sequence divergence than most coding regions, and the divergent lengths of noncoding regions affected chloroplast genome size variation among species. Almost all the chloroplastic protein-coding genes bias ended with $\mathrm{A} /$ U. Mutations and selection forces, particularly natural selection, shaped the codon bias pattern of most genes. Most of these mentioned genes were predicted to have a signature of purifying selection, whereas photosynthetic genes experienced a relatively relaxed purifying selection, whose codon bias and evolutionary rate were also driven by other factors such as gene expression level and gene length. Additionally, chloroplast genomes sequenced in this study and those simultaneously sequenced did have differences in both gene content and structure, which brought different results of quadripartite boundaries, substitution rates, and selective pressure. Nevertheless, chloroplast genomes sequenced together with the ones sequenced simultaneously can provide insights into both the genetic relationships among Sect. Marmorata and the other species of Paris. Moreover, the results aid to expand the current understanding of the evolutionary history of Paris, particularly Sect. Marmorata.

\section{Methods}

Taxon sampling, DNA extraction and sequencing

$P$. marmorata and $P$. luquanensis analyzed in this study were cultivated and collected in green house (Kunming) of Xishuangbanna Tropical Botanical Garden. No specific permits are required for sampling. Total genomic DNA was extracted from $100 \mathrm{mg}$ of fresh healthy leaves using a modified CTAB method 
$[52,53]$, and the quality of each sample was assessed by agarose gel electrophoresis. The whole chloroplast genomes were amplified using long-range PCR and nine universal primer pairs according to the procedure outlined by Yang et al [53]. Then, six $\mu \mathrm{g}$ of purified PCR products was mixed and fragmented to construct short insert libraries (measuring 200-500 bp in length) according to the procedures outlined in the Illumina manual. The paired-end libraries were then sequenced using Illumina MiSeq 2000 platform (Illumina, San Diego, CA, USA) at Germplasm Bank of Wild Species, Kunming Institute of Botany, Chinese Academy of Sciences.

\section{Assembly and annotation}

Raw reads were filtered with the quality control program NGSQCToolkit v2.3.3 to obtain high quality Illumina data (the cut-off value for percentage of read length was 80, and the cut-off value for PHRED quality score was 30) and adaptor-free reads. Filtered reads were then assembled into contigs using SPAdes v3.6.1. Outputted contigs were aligned with the reference $P$. polyphylla var. yunnanensis chloroplast genome (Genbank accession No. KT805945) Contigs were then aligned with the reference genome to assemble each chloroplast genome sequence using Geneious v4.8.4. Assembled genome sequences were annotated using the online tool DOGMA and Geneious v4.8.4 [54, 55], and then annotated sequences were manually edited for start and stop codons. All tRNA genes were further confirmed by the online tRNAscan-SE search server [56]. The annotated chloroplast genomes were deposited in GenBank with accession numbers: P. marmorata (MF495705) and P. luquanensis (MF417768). The annotated GenBank files of the two Paris chloroplast genomes were uploaded to obtain gene maps using the online tool GenomeVx [57].

\section{Repeat sequence identification}

Repeat elements in chloroplast genomes of $P$. marmorata and $P$. luquanensis were investigated using three different programs. The position and type of SSR were ascertained using the microsatellite identification tool MISA v1.0 [58], and each repeat sequence length was screened to be $\geq 10 \mathrm{bp}$. SSRs were identified with thresholds of $10,5,4,3,3$, and 3 repeat units for mono-, di-, tri-, tetra-, penta-, and hexa-nucleotides, respectively. Diversity of chloroplast SSR markers were further estimated using the Shannon-Winener index and PIC. Tandem repeat sequences ( $>10 \mathrm{bp}$ in length) were identified with TRF v4.09 [59], with parameters of 2, 7, and 7 for matches, mismatches and indels, respectively. The minimum alignment score and maximum period size were set to 50 and 500, respectively. Meanwhile, the size and location of both forward and inverted/palindromic repeats were determined using REPuter v1.0 [60]. The parameters were set with a minimal repeat size of $30 \mathrm{bp}$, hamming distance of 3 $\mathrm{kb}$, and $90 \%$ sequence identity threshold. Gene and intergenic spacer regions harbored repeat sequence were extracted, on the basis of the loci of repeats. These regions were then applied to infer phylogenetic relationships with Neighbor-Joining algorithm in MEGA v6.06 and Maximum Likelihood algorithm of RAxML v7.2.6.

\section{Comparison of chloroplast genome sequences}

To investigate the sequence divergence among Sect. Marmorata chloroplast genomes, several released chloroplast genomes were retrieved from NCBI: $P$. marmorata (NC_033516, denoted by PMa), P. luquanensis (NC_033514, denoted by PLa), and P. verticillata (NC_024560, denoted by PV). Four chloroplast genome sequences of Sect. Marmorata were aligned using MAFFT v7.305b [61] and were manually adjusted using Se-Al v2.0 [62]. A sliding window analysis was conducted to compare $\pi$ among the chloroplast genomes of Sect. Marmorata, using DnaSP v5.0 [63]. The window length was $600 \mathrm{bp}$ with a $200 \mathrm{bp}$ step size. To reveal both inter- and intra-specific variations, the full alignments of chloroplast genome sequences of Sect. Marmorata species and $P$. verticillata were visualized with Shuffle-LAGAN mode in mVISTA program [64].

\section{Codon usage and substitution rate calculation}

RSCU, GC3s, and ENc for 71 protein-coding genes were calculated using CodonW v1.4.4 [65]. Then, the relationships between ENc and GC3s were analyzed. $K_{\mathrm{a}}, K_{\mathrm{s}}$ and their ratios $K_{\mathrm{a}} / K_{\mathrm{s}}$ were estimated with ParaAT v2.0 and KaKs_caculator v2.0 [66, 67]. These substitution analyses of 77 conserved protein-coding genes from chloroplast genomes of Sect. Marmorata species were implemented, using alignments with $P$. verticillata. Boxplots were constructed for each functional category/group, and plotted with SigmaPlot v13.0.

\section{Additional files}

\footnotetext{
Additional file 1: Table S1. Summary of chloroplast genome characteristics of Sect. Marmorata. Table S2. List of gene function in the chloroplast genomes. Table S3. Distribution of tandem repeats from the chloroplast genomes. Table S4. Distribution of dispersed, forward and inverted/palindromic repeats from chloroplast genomes. Table S5. Type and number of SSRs in the chloroplast genomes. (PDF 396 kb)

Additional file 2: Figure S1. Venn diagram analysis for gene composition from chloroplast genomes of Sect. Marmorata via different assembly methods and reference genomes. Figure S2. Six kinds of SSR motifs in fifteen Paris chloroplast genomes. P. polyphylla var. yunnanensisO was sequenced by Song et al (2015). P. marmorata0, P. luquanensis0, P. polyphylla var. yunnanensis were sequenced by Huang et al (2016). P.
} 
marmorata and $P$. luquanensis were sequenced in this study. Figure S3. Phylogenetic trees of genes spacer regions harbored repeat sequence, using $\mathrm{NJ}$ (bootstrap values on the left of slashes) and $\mathrm{ML}$ (bootstrap values on the right of slashes) algorithms. Figure S4. The $K_{\mathrm{a}} / K_{\mathrm{s}}$ ratios of 71 protein-coding genes from chloroplast genome of Sect. Marmorata. Red bars and blue bars denote $P$. marmorata (PMa) and $P$. luquanensis (PLa) sequenced previously; green bars and yellow bars denote $P$. marmorata (PMb) and $P$. luquanensis (PLb) sequenced in this study. Figure S5. Comparison of $K_{a}, K_{s}$, and $K_{a} / K_{s}$ ratios of Sect. Marmorata chloroplast genes. (A-C) denote $K_{\mathrm{a}}, K_{\mathrm{s}}$ and $K_{\mathrm{a}} / K_{\mathrm{s}}$, respectively. Red highlight boxplots indicate photosynthesis genes, green ones indicate genes involved in selfreplication, and blue ones indicate other genes. SR: small subunit of ribosome, LR: large subunit of ribosome, DR: DNA-dependent RNA, TF: translational initiation factor, ND: NAPH dehydrogenase, PI: photosystem I, PII: photosystem II, CC: cytochrome b/f complex, AS: ATP synthase gene, LS: large subunit of rubisco, SA: subunit of acetyl-CoA, CS: cytochrome synthesis, CT: c-type cytochrome synthesis, PR: protease, MA: maturase, CO: conserved ORF. (PDF $793 \mathrm{~kb}$ )

Additional file 3: Table S6. Number of SSRs in fifteen chloroplast genomes of Paris species. Table S7. Polymorphism of SSRs in chloroplast genomes of Sect. Marmorata. (XLSX 17 kb)

\section{Abbreviations}

CDS: Coding DNA sequence; CNS: Conserved noncoding sequence; DOGMA: Dual Organellar GenoMe Annotator; ENc: Effective number of codons; GC3s: GC content on the third synonymous codon position; IR: Inverted repeat; $K_{a}$ : Nonsynonymous substitutions per non-synonymous site; $K_{s}$ : Synonymous substitutions per synonymous site; LSC: Large single copy; PIC: Polymorphic information content; RSCU: Relative synonymous codon usage; SNP: Single nucleotide polymorphism; SSC: Small single copy; SSR: Simple sequence repeat; TRF: Tandem Repeats Finder; $\pi$ : Nucleotide diversity

\section{Acknowledgments}

We are very grateful to Junbo Yang, Zhirong Zhang and Zhanshan He (Germplasm Bank of Wild Species in Kunming Institute of Botany) for their help with experiments and data analyses. We also thank the Public Technology Service Center at Xishuangbanna Tropical Botanical Garden for providing the computer resources.

\section{Funding}

This research was supported by National Natural Science Foundation of China (No. 31800273, 31471220, 91440113); Start-up Fund from Xishuangbanna Tropical Botanical Garden; Top Talents Program in Science and Technology' from Yunnan Province; the CAS "Light of West China" Program. The funders had no role in study design, data collection, analysis and interpretation, or preparation of the manuscript. Publication costs are funded by National Natural Science Foundation of China (No. 31800273), the CAS "Light of West China" Program.

\section{Availability of data and materials}

The chloroplast genomes generated during the current study were deposited in NCBI with accession number MF495705 (P. marmorata) and MF417768 (P. luquanensis).

\section{About this supplement}

This article has been published as part of BMC Genomics Volume 19 Supplement 10, 2018: Proceedings of the 29th International Conference on Genome Informatics (GIW 2018): genomics. The full contents of the supplement are available online at https://bmcgenomics.biomedcentral.com/articles/ supplements/volume-19-supplement-10.

\section{Authors' contributions}

$C N L$ and $X Y G$ designed the experiments. $X Z$, JL performed the experiments. $X Y G, X Z, D Z$ analyzed data. XYG, XZ, HHM wrote the paper. All authors reviewed and approved the manuscript.

Ethics approval and consent to participate Not applicable.

\section{Consent for publication}

Not applicable.

\section{Competing interests}

The authors declare that they have no competing interests.

\section{Publisher's Note}

Springer Nature remains neutral with regard to jurisdictional claims in published maps and institutional affiliations.

\section{Author details}

${ }^{1}$ CAS Key Laboratory of Tropical Plant Resources and Sustainable Use, Xishuangbanna Tropical Botanical Garden, Chinese Academy of Science, Menglun 666303, Yunnan, China. ${ }^{2}$ Center for Integrative Conservation, Xishuangbanna Tropical Botanical Garden, Chinese Academy of Sciences, Kunming 650223, Yunnan, China. ${ }^{3}$ University of Chinese Academy of Sciences, Beijing 100049, China.

Published: 31 December 2018

\section{References}

1. Gad HA, El-Ahmady SH, Abou-Shoer MI, Al-Azizi MM. Application of chemometrics in authentication of herbal medicines: a review. Phytochem Anal. 2013;24(1):1-24.

2. Li H. The genus Paris L. 2nd ed. Beijing: Science Press; 2008.

3. Cheng ZX, Liu BR, Qian XP, Ding YT, Hu WJ, Sun J, et al. Proteomic analysis of anti-tumor effects by Rhizoma Paridis total saponin treatment in HepG2 cells. J Ethnopharmacol. 2008;120(2):129-37.

4. Yan L, Zhang Y, Gao W, Man S, Wang Y. In vitro and in vivo anticancer activity of steroid saponins of Paris polyphylla var. yunnanensis. Exp Oncol. 2009;31(1):27-32.

5. Man SL, Gao WY, Zhang YJ, Wang JY, Zhao WS, Huang LQ, et al. Qualitative and quantitative determination of major saponins in Paris and Trillium by HPLC-ELSD and HPLC-MS/MS. J Chromatogr B. 2010;878(29):2943-8.

6. Timmis JN, Ayliffe MA, Huang CY, Martin W. Endosymbiotic gene transfer: organelle genomes forge eukaryotic chromosomes. Nat Rev Genet. 2004;5(2):123-35.

7. Liu J, Qi ZC, Zhao YP, Fu CX, Xiang QY. Complete cpDNA genome sequence of Smilax china and phylogenetic placement of Lilialesinfluences of gene partitions and taxon sampling. Mol Phylogenet Evol. 2012:64(3):545-56.

8. Raubeson LA, Jansen RK. Chloroplast genomes of plants. 1st ed. Cambridge: CABI press; 2005.

9. Jansen RK, Raubeson LA, Boore LA, Chumley TW, Haberle RC, Wyman SK, et al. Methods for obtaining and analyzing whole chloroplast genome sequences. Method Enzymol. 2005;395:348-84

10. Wolfe KH, Li WH, Sharp PM. Rates of nucleotide substitution vary greatly among plant mitochondrial, chloroplast, and nuclear DNAs. Proc Natl Acad Sci U S A. 1987;84(24):9054-8.

11. Provan J, Powell W, Hollingsworth PM. Chloroplast microsatellites: new tools for studies in plant ecology and evolution. Trends Ecol Evol. 2001; 16(3):142-7.

12. Ravi V, Khurana J, Tyagi A, Khurana P. An update on chloroplast genomes. Plant Syst Evol. 2008;271:101-22.

13. Powell W, Morgante M, Andre C, McNicol JW, Machray GC, Dyle JJ, et al. Hypervariable microsatellites provide a general source of polymorphic DNA markers for the chloroplast genome. Curr Biol. 1995;5(9):1023-9.

14. Haasl RJ, Payseur BA. Multi-locus inference of population structure: a comparison between single nucleotide polymorphisms and microsatellites. Heredity. 2011;106(1):158-71.

15. Delplancke M, Alvarez N, Espindola A, Joly H, Benoit L, Brouck E, et al. Gene flow among wild and domesticated almond species: insights from chloroplast and nuclear markers. Evol Appl. 2012;5(4):317-29.

16. Allender CJ, King GJ. Origins of the amphiploid species Brassica napus L. investigated by chloroplast and nuclear molecular markers. BMC Plant Biol. 2010;10:54

17. Dempewolf H, Kane NC, Ostevik KL, Geleta M, Barker MS, Lai Z, et al. Establishing genomic tools and resources for Guizotia abyssinica (If) Cass.-the development of a library of expressed sequence tags, microsatellite loci, and the sequencing of its chloroplast genome. Mol. Ecol Res. 2010;10(6):1048-58. 
18. Sonah H, Deshmukh RK, Sharma A, Singh VP, Gupta DK, Gacche RN, et al. Genome-wide distribution and organization of microsatellites in plants: an insight into marker development in Brachypodium. PLoS One. 2011:6(6):e21298.

19. Wheeler GL, Dorman HE, Buchanan A, Challagundla L, Wallace LE. A review of the prevalence, utility, and caveats of using chloroplast simple sequence repeats for studies of plant biology. Appl Plant Sci. 2014;2(12):apps.1400059.

20. Huang YL, Li XJ, Yang ZY, Yang CJ, Yang JB, Ji YH. Analysis of complete chloroplast genome sequences improves phylogenetic resolution in Paris (Melanthiaceae). Front Plant Sci. 2016;7:1797.

21. Sugita M, Sugiura M. Regulation of gene expression in chloroplasts of higher plants. Plant Mol Biol. 1996;32(1-2):315-26.

22. Rouwendal GJ, Mendes O, Wolbert EJ, Douwe De Boer A. Enhanced expression in tobacco of the gene encoding green fluorescent protein by modification of its codon usage. Plant Mol Biol. 1997:33(6):989-99.

23. Morton BR. The role of context-dependent mutations in generating compositional and codon usage bias in grass chloroplast DNA. J Mol Evol. 2003;56(5):616-29.

24. Ogihara Y, Terachi T, Sasakuma T. Intramolecular recombination of chloroplast genome mediated by short direct-repeat sequences in wheat species. Proc Natl Acad Sci U S A. 1988;85(22):8573-7.

25. Milligan BG, Hampton JN, Palmer JD. Dispersed repeats and structural reorganization in subclover chloroplast DNA. Mol Biol Evol. 1989;6(4):355-68.

26. Curci PL, Paola DD, Danzi D, Vendramin GG, Sonnante G. Complete chloroplast genome of the multifunctional crop globe artichoke and comparison with other Asteraceae. PLoS One. 2015;10(3):e0120589.

27. Redwan R, Saidin A, Kumar S. Complete chloroplast genome sequence of MD-2 pineapple and its comparative analysis among nine other plants from the subclass Commelinidae. BMC Plant Biol. 2015;15:196.

28. Vendramin GG, Degen B, Petit RJ, Anzidei M, Madaghiele A, Ziegenhagen B. High level of variation at Abies alba chloroplast microsatellite loci in Europe. Mol Ecol. 1999;8(7):1117-26.

29. Deguilloux MF, Pemonge MH, Petit RJ. Use of chloroplast microsatellites to differentiate oak populations. Ann For Sci. 2004;61(8):825-30.

30. Gaudeul M, Giraud T, Kiss L, Shykoff JA. Nuclear and chloroplast microsatellites show multiple introductions in the worldwide invasion history of common ragweed, Ambrosia artemisiifolia. PloS One. 2011; 6(3):e17658.

31. Piya S, Nepal MP. Characterization of nuclear and chloroplast microsatellite markers for Falcaria vulgaris (Apiaceae). Am J Plant Sci. 2013:4:590-5.

32. Kim KJ, Lee HL. Complete chloroplast genome sequences from Korean ginseng (Panax schinseng Nees) and comparative analysis of sequence evolution among 17 vascular plants. DNA Res. 2004;11(4):247-61.

33. Huotari $\mathrm{T}$, Korpelainen $\mathrm{H}$. Complete chloroplast genome sequence of Elodea canadensis and comparative analyses with other monocot plastid genomes. Gene. 2012;508(1):96-105.

34. Yi DK, Lee HL, Sun BY, Chung MY, Kim KJ. The complete chloroplast DNA sequence of Eleutherococcus senticosus (Araliaceae); comparative evolutionary analyses with other three asterids. Mol Cells. 2012;33(5):497-508.

35. Shaw J, Lickey EB, Schilling EE, Small RL. Comparison of whole chloroplast genome sequences to choose noncoding regions for phylogenetic studies in angiosperms: the tortoise and the hare III. Am J Bot. 2007;94(3):275-88.

36. Worberg A, Quandt D, Barniske AM, Lohne C, Hilu KW, Borsch T. Phylogeny of basal eudicots: insights from non-coding and rapidly evolving DNA. Org Divers Evol. 2007;7(1):55-77.

37. Khakhlova O, Bock R. Elimination of deleterious mutations in plastid genomes by gene conversion. Plant J. 2006;46(1):85-94.

38. Zhang $\mathrm{H}$, Li C, Miao H, Xiong S. Insights from the complete chloroplast genome into the evolution of Sesamum indicum L. PLoS One. 2013;8(11):e80508.

39. Dong W, Xu C, Cheng T, Zhou S. Complete chloroplast genome of Sedum sarmentosum and chloroplast genome evolution in Saxifragales. PLoS One. 2013;8(10):e77965.

40. Kim SC, Kim JS, Kim JH. Insight into infrageneric circumscription through complete chloroplast genome sequences of two Trillium species. AoB Plants. 2016;8:plw015.

41. Raubeson LA, Peery R, Chumley TW, Dziubek C, Fourcade HM, Boore JL, et al. Comparative chloroplast genomics: analyses including new sequences from the angiosperms Nuphar advena and Ranunculus macranthus. BMC Genomics. 2007:8:174.
42. Gao L, Yi X, Yang YX, Su YJ, Wang T. Complete chloroplast genome sequence of a tree fern Alsophila spinulosa: insights into evolutionary changes in fern chloroplast genomes. BMC Evol Biol. 2009;9:130.

43. Yang M, Zhang XW, Liu GM, Yin YX, Chen KF, Yun QZ, et al. The complete chloroplast genome sequence of date palm (Phoenix dactylifera L). PLoS One. 2010;5(9):e12762.

44. Wright $F$. The 'effective number of codons' used in a gene. Gene. 1990;87(1):23-9.

45. Sablok G, Nayak KC, Vazquez F, Tatarinova TV. Synonymous codon usage, GC3, and evolutionary patterns across plastomes of three pooid model species: emerging grass genome models for monocots. Mol Biotechnol. 2011:49(2):116-28.

46. Yang Z, Nielsen R. Estimating synonymous and nonsynonymous substitution rates under realistic evolutionary models. Mol Ecol Evol. 2000;17(1):32-43.

47. Xu JH, Liu Q, Hu W, Wang T, Xue Q, Messing J. Dynamics of chloroplast genomes in green plants. Genomics. 2015;106(4):221-31.

48. Muse SV, Gaut BS. Comparing patterns of nucleotide substitution rates among chloroplast loci using the relative ratio test. Genetics. 1997;146(1):393-9.

49. Mcinerney JO. The causes of protein evolutionary rate variation. Trends Ecol Evol. 2006;21(5):230-2.

50. Wang Z, Zhang J. Why is the correlation between gene importance and gene evolutionary rate so weak? PLoS Genet. 2009;5(1):e1000329.

51. Alvarez-Ponce D, Fares MA. Evolutionary rate and duplicability in the Arabidopsis thaliana protein-protein interaction network. Genome Biol Evol. 2012:4(12):1263-74.

52. Dolye JJ, Doyle JL. A rapid DNA isolation procedure for small quantities of fresh leaf tissue. Phytochem Bull. 1987;19:11-5.

53. Yang JB, Li DZ, Li HT. Highly effective sequencing whole chloroplast genomes of angiosperms by nine novel universal primer pairs. Mol Ecol Resour. 2014;14(5):1024-31.

54. Wyman SK, Jansen RK, Boore JL. Automatic annotation of organellar genomes with DOGMA. Bioinformatics. 2004;20(17):3252-5.

55. Kearse M, Moir R, Wilson A, Stones-Havas S, Cheung M, Sturrock S, et al. Geneious basic: an integrated and extendable desktop software platform for the organization and analysis of sequence data. Bioinformatics. 2012;28(12):1647-9.

56. Lowe TM, Eddy SR. tRNAscan-SE: a program for improved detection of transfer RNA genes in genomic sequence. Nucleic Acids Res. 1997;25(5):955-64.

57. Conant GC, Wolfe KH. GenomeVx: simple web-based creation of editable circular chromosome maps. Bioinformatics. 2008;24(6):861-2.

58. Thiel T, Michalek W, Varshney RK, Graner A. Exploiting EST databases for the development and characterization of gene-derived SSR-markers in barley (Hordeum vulgare L.). Theor Appl Genet. 2003;106(3):411-22.

59. Benson $G$. Tandem repeats finder: a program to analyze DNA sequences. Nucleic Acids Res. 1999;27(2):573-80.

60. Kurtz SJ, Choudhuri JV, Ohlebusch E, Schleiermacher C, Stoye J, Giegerich R. REPuter: the manifold applications of repeat analysis on a genomic scale. Nucleic Acids Res. 2001;29(22):4633-42.

61. Katoh K. Standley DM. MAFFT multiple sequence alignment software version 7: improvements in performance and usability. Mol Biol Evol. 2013; 30(4):772-80.

62. Rambaut A. 1996. Se-Al: Sequence Alignment Editor. version 2.0. http://tree. bio.ed.ac.uk/software/seal/

63. Librado P, Rozas J. DnaSP v5: a software for comprehensive analysis of DNA polymorphism data. Bioinformatics. 2009;25(11):1451-2.

64. Frazer KA, Pachter L, Poliakov A, Rubin EM, Dubchak IVISTA. Computational tools for comparative genomics. Nucleic Acids Res. 2004;32:W273-9.

65. Peden JF. Analysis of codon usage. PhD thesis. UK: University of Nottingham; 1999

66. Zhang Z, Li J, Zhao XQ, Wang J, Wong GKS, KaKs YJ. Calculator: calculating $\mathrm{Ka}$ and Ks through model selection and model averaging. Genom Proteom Bioinformatics. 2006;4(4):259-63.

67. Zhang Z, Xiao JF, Wu JY, Zhang HY, Liu GM, Wang XM, et al. ParaAT: a parallel tool for constructing multiple protein-coding DNA alignments. Biochem Bioph Res Co. 2012;419(4):779-81. 